\title{
Comparación de la función pulmonar de pacientes con diabetes mellitus tipo 2 sometidos a tratamiento de insulina inyectada versus tratamiento con hipoglucemiantes orales
}

\author{
Comparison of the pulmonary function of patients with type \\ 2 diabetes mellitus treated with insulin injections versus that \\ of patients treated with oral hypoglucemic agents
}

\author{
Héctor F. Restrepo, Martín Rondón, María X. Rojas • Bogotá, D.C. \\ Yolanda Torres • Medellín. Pablo Aschner, Rodolfo J. Dennis • Bogotá, D.C.
}

\section{Resumen}

Introducción: la potencial asociación entre el tipo de tratamiento de la diabetes mellitus tipo 2 (DM2) y alteración de la función pulmonar es algo poco estudiado hasta ahora.

Objetivos: comparar la función pulmonar de pacientes con DM2 que reciben tratamiento con insulina inyectable versus hipoglicemiantes orceles (HO). Determinar si niveles de marcadores de inflamación en pacientes con tratamiento basado en insulina son diferentes a los de los tratados con HO.

Métodos: estudio observacional analítico de corte transversal a partir de una muestra de conveniencia de 369 pacientes con diagnóstico de DM2, y tratamiento con insulina o HO. Se realizaron espirometrías, y se obtuvieron valores residuales promedios para VEF1, CVF y relación VEF1/CVF. Mediante regresión lineal múltiple, se ajustó por diferencias en determinantes conocidos de la función pulmonar, así como por control de la diabetes y tiempo desde el diagnóstico. Adicionalmente, se midieron niveles de marcadores inflamatorios sanguíneos para cada grupo de tratamiento.

Resultados: 63 pacientes (17\%) recibían tratamiento con insulina y 306 (83\%) con HO. La diferencia en residuales favoreció a los tratados con HO. Para VEF1, CVF y VEF1/CVF la diferencia fue 57.6 $\mathrm{mL}$ (IC95\% 32.45-82.74; P 0.0047), 45.6 mL (IC95\% 20.84-70.39; P 0.0231) y 0.017, (IC95\% 0.01$0.02, \mathrm{P}<0.0001)$, respectivamente. No hubo cambios estadísticamente significativos en marcadores de inflamación.

Conclusiones: los pacientes en tratamiento con $\mathrm{HO}$ presentaron mejor función pulmonar que los tratados con insulina. Este hallazgo de diferencias en función pulmonar pudiera tener implicación clínica en el manejo de los pacientes diabéticos, pero debe confirmarse en estudios prospectivos (Acta Med Colom; 2010: 35: 113-118).

Palabras clave: diabetes mellitus, función pulmonar, pronóstico, riesgo, espirometría.

\footnotetext{
Abstract

Introduction: the potential association between the type of treatment of type 2 diabetes mellitus (DM2) and impaired lung function is something rarely studied so far.

Objectives: to compare the lung function of patients with DM2 who are treated with injectable insulin versus HO. To determine whether levels of inflammatory markers in patients with insulin-based treatment are different from those treated with HO.

Methods: an observational, analytical, cross-sectional study from a convenience sample of 369 patients diagnosed with DM2 and treated with insulin or HO. Spirometry was performed, and residual values were averaged for FEV1, FVC and FEV1/FVC ratios. Multiple linear regression results were adjusted by differences in known determinants of lung function, as well as control of diabetes and time since diagnosis. Additionally, we measured blood levels of inflammatory markers for each treatment group.
}

Dr. Héctor F. Restrepo, MDVZ: MSc Departamentos de Investigaciones y Medicina Interna, Fundación Cardioinfantil - Instituto de Cardiología, Bogotá; Dres. Martín Rondón, MSc y María X. Rojas RN, MSc: Departamento de Epidemiología Clínica y Bioestadística, Facultad de Medicina Universidad Javeriana, Bogotá, D.C.; Dra. Yolanda Torres Galvis MD, MSc: Universidad CES, Medellín; Dr. Pablo Aschner, MD MSc: Asociación Colombiana de Diabetes, Bogotá, D.C.; Dr. Rodolfo J. Dennis, MD MSc: Departamentos de Investigaciones y Medicina Interna, Fundación Cardioinfantil - Instituto de Cardiología, Departamento de Epidemiología Clínica y Bioestadística, Facultad de Medicina, Universidad Javeriana, Bogotá, D. C. Todos en Colombia.

Correspondencia. Dr. Rodolfo Dennis, MD, MSc. Departamento de Investigaciones, Fundación Cardioinfantil. Carrera 13B No. 163-85, 3er Piso, Fax: +6793505. Bogotá, Colombia.

E-mail: rdennis@cardioinfantil.org Recibido: 01/VII//10 Aceptado: 09/IX/10 
Results: 63 patients (17\%) were treated with insulin and $306(83 \%)$ with $\mathrm{OH}$. The difference in residual favored those treated with $\mathrm{HO}$. For FEV1, FVC and FEV1/FVC the difference was $57.6 \mathrm{~mL}$ (95\% CI 32.45 to $82.74, \mathrm{P} 0.0047$ ), $45.6 \mathrm{~mL}$ (95\% CI 20.84 to $70.39, \mathrm{P} 0.0231$ ) and 0.017 (95\% CI 0.01 to $0.02, \mathrm{P}<0.0001)$, respectively. There were no statistically significant changes in inflammation markers.

Conclusions: patients treated with $\mathrm{HO}$ showed better lung function than those treated with insulin. This finding of differences in lung function may have clinical implications in the management of diabetic patients, but needs to be confirmed in prospective studies (Acta Med Colom; 2010: 35: 113-118).

Keywords: diabetes mellitus, pulmonary function, prognosis, risk, spirometry.

\section{Introducción}

Un aspecto ampliamente estudiado de la diabetes mellitus (DM), tanto tipo 1 como tipo 2, es su posible asociación con menor función pulmonar, medida por fuertes determinantes de incapacidad y de muerte por causa pulmonar, como el volumen espiratorio forzado en un segundo (VEF1) o la capacidad vital forzada (CVF) (1-4).

De acuerdo con una revisión sistemática reciente publicada por Borst y col (5), la inflamación, microangiopatía, y alteraciones en las proteínas pulmonares, pueden tener todas un rol importante en el deterioro de la función pulmonar visto en la diabetes. El primer mecanismo tiende a comparar el deterioro observado en los pacientes con enfisema con el que se observa en la diabetes y las enfermedades cardiovasculares. Tanto la DM2, la enfermedad pulmonar crónica, como la enfermedad pulmonar obstructiva crónica (EPOC), se han asociado con mayores niveles de mediadores y marcadores inflamatorios, y respuesta inflamatoria sistémica crónica (6-10). Estudios en ratas han mostrado que la inflamación de las vías aéreas se caracteriza por una elevación significativa de eosinófilos y neutrófilos (7).

Un aspecto poco estudiado hasta el momento, es el rol que puede tener el tratamiento de la DM2 en el deterioro de la función pulmonar. De igual forma, poco se ha especulado hasta ahora, si en pacientes diabéticos existe asociación entre marcadores de inflamación sistémica, la función pulmonar, y el tratamiento con insulina o con hipoglicemiantes orales (HO). Aunque se ha descrito una vía causal por la cual la insulina pudiese asociarse con deterioro (11), o con mejoría (12) de la función pulmonar, puede también existir un efecto benéfico con el uso de $\mathrm{HO}$, especialmente con el uso de sensibilizantes a la insulina, tipo metformina o las tiazolidinedionas (13). Sin embargo, otros factores condicionantes del tratamiento tales como duración de la diabetes, el control glucémico y la resistencia a la insulina en sujetos diabéticos pudiesen explicar los cambios que estos pacientes muestran en la función pulmonar, además de otros factores de riesgo como el tabaquismo y la obesidad (14-18). Todos estos factores pueden actuar sinérgicamente en el diabético y al ser variables modificadoras de efecto, podrían confundir una eventual asociación entre tratamiento y deterioro o mejoría de la función pulmonar.

Con la hipótesis que las diferencias encontradas en la función pulmonar de los sujetos con DM2 pueden ser ex- plicadas por diferencias en el tipo de tratamiento recibido para control de la diabetes, se realizó el presente análisis exploratorio en el cual se compara la función pulmonar de sujetos con DM2 y tratados con insulina con la función pulmonar de sujetos con DM2 tratados con $\mathrm{HO}$, ajustando por potenciales variables de confusión o modificadoras del efecto. Para esto se estudia una población de pacientes con DM2 que asistieron a la Asociación Colombiana de Diabetes (ACD) en un periodo de dos años e hicieron parte de un estudio previo cuyos métodos ya se encuentran publicados (18), y que tuvo como objetivo comparar la función pulmonar en sujetos con y sin DM2. Brevemente, dicho estudio incluyó 495 hombres y mujeres, entre 35 y 65 años de edad, con diagnóstico médico de DM2 de acuerdo con la definición dada por la Asociación Latinoamericana de Diabetes ALAD (glucemia casual igual o mayor a $200 \mathrm{mg} / \mathrm{dL}$, o una glucemia en ayunas igual o mayor a $126 \mathrm{mg} / \mathrm{dL}$, o con una glucemia igual o mayor a $200 \mathrm{mg} / \mathrm{dL}$ dos horas después de una carga de glucosa en una prueba de tolerancia oral a la glucosa) (19), que fueron seleccionados a través de un muestreo no probabilístico e incluyó todos los casos consecutivos de DM2 que consultaron a la ACD entre julio de 2005 y septiembre de 2007. A estos sujetos se les realizaron mediciones en sangre de glucemia, hemoglobina glicosilada y marcadores de inflamación [proteína $\mathrm{C}$ reactiva ultrasensible (PCR), ferritina, fibrinógeno, interleukina 6 (IL6) y factor de necrosis tumoral (TNF-alfa)], así como de la función pulmonar por curva de flujo volumen. Se excluyeron de la muestra de estudio los pacientes que teniendo diagnóstico de DM cumplieron con alguno de los siguientes criterios: diagnóstico previo de artritis reumatoide u otra enfermedad del colágeno; de traqueomalacia, fibrosis quística, cáncer de pulmón, tuberculosis pulmonar, cirugías del tórax, corazón, o pulmón; antecedentes de herida o traumatismo en el tórax, procedimientos como pleurodesis o toracostomías; embarazo y deformidades de la boca u otra limitante (traqueotomía) para realizar apropiadamente las pruebas de función pulmonar. Los resultados mostraron que los sujetos con DM2 e inadecuado control tuvieron menor CVF y el VEF 1 de lo predicho y que la de los sujetos con control adecuado (18).

Finalmente, se presenta la distribución de los niveles en sangre de sustancias proinflamatorias de baja intensidad en los diabéticos con tratamiento de insulina y en los tratados con HO. 


\section{Métodos}

Estudio observacional analítico de corte transversal donde se incluyeron 369 pacientes con diagnóstico de DM2 tratados con insulina o HO. Para determinar los grupos de comparación, se definió como expuesto a insulina, aquellas personas que cumpliendo la definición de DM2, informaran que al momento de ingreso al estudio recibían insulina inyectada subcutánea como único tratamiento hipoglucemiante actual. De igual forma, se definió como expuesto a $\mathrm{HO}$, a aquellos sujetos con DM2 que estuviesen recibiendo cualquier tratamiento hipoglucemiante oral, tanto secretagogos (ej. glibenclamida) como sensibilizantes (ej. metformina), sin exposición actual a insulina. No se recogió información sobre tiempo de uso con uno o con otro hipoglucemiante.

A los pacientes elegibles por encuesta, se les realizaron mediciones de glucemia, HbAlc, y de curva de flujo-volumen, y se documentó el tipo de tratamiento que estaban recibiendo en ese momento. El control de calidad de las mediciones espirométricas, incluyó el seguimiento de las recomendaciones dadas por la American Toracic Society (ATS) (20), y la verificación periódica de la exactitud en la medición, usando jeringa de 8 litros diseñada especialmente para esta calibración.

Para los análisis comparativos ajustados, las variables dependientes fueron el volumen espiratorio forzado en un segundo (VEF1), la capacidad vital forzada (CVF), y la relación VEF1/CVF. La independiente de interés el tratamiento de la diabetes. Las variables independientes de ajuste o con intervinientes fueron edad, sexo, talla, tabaquismo, tiempo desde el diagnostico de la diabetes, niveles de HbAlc y niveles en sangre de proteína $C$ reactiva ultrasensible (PCR), ferritina, fibrinógeno, interleukina 6 (IL6) y factor de necrosis tumoral (TNF-alfa). Se definió "mal control diabético" como pacientes con hemoglobina glucosilada mayor de $7 \%$ (21).

\section{Manejo y procesamiento de muestras}

Las muestras de sangre fueron tomadas después de un mínimo de seis horas de ayuno (con excepción de los diabéticos insulinodependientes), utilizando tubos de vacío, se centrifugaron y procesaron para los niveles de glucosa en ayunas, HbA1c, fibrinógeno y ferritina, siguiendo procedimientos estándar de laboratorio. Un conjunto de muestras de suero fueron almacenadas a $-20^{\circ} \mathrm{C}$ durante un máximo de dos meses para los otros análisis, TNF- $\alpha$, IL-6, y PCR de alta sensibilidad las cuales fueron medidas con un método inmunométrico enzimático secuencial en fase sólida por quimioluminiscencia, utilizando el analizador IMMULITE 1000 (EURO / DPC Ltd, Llanberis, Reino Unido). Cada ejecución de PCR, TNF e IL-6 se procesaron por duplicado, y se cumplió con el estándar de control de ensayos específicos proporcionados por el fabricante, dos por el TNF- $\alpha$ e IL-6 (alta y baja), y tres para el PCR (alta, intermedia y baja).

\section{Análisis estadístico}

Se diseñó una base para captura de los datos en el programa ACCES/ Office 2000, y los análisis estadísticos se realizaron en el programa STATA versión 10.0. Se obtuvieron inicialmente las distribuciones de frecuencia simples que permitían identificar variables que pudiesen requerir transformación para su manejo paramétrico $u$ otro tipo de análisis. Las diferencias estadísticas entre grupos de tratamiento con respecto a variables categóricas (tabaquismo, antecedentes patológicos) fueron evaluadas mediante la distribución del $\chi^{2}$, y las continuas $\left(\mathrm{VEF}_{1}\right.$, CVF) mediante pruebas para diferencias entre promedios (test T). La evaluación de la función pulmonar se realizó a partir del valor de la diferencia (residual) entre el valor observado del $\mathrm{VEF}_{1}$, la CVF, y la relación $\mathrm{VEF}_{1} / \mathrm{CVF}$, y del valor predicho. Este último valor fue estimado a partir de las ecuaciones propuestas por Hankinson (22) para mexicoamericanos, que ha mostrado tener mejor ajuste para la población adulta de Bogotá (23) que otras ecuaciones (24). Para evaluar la relación entre los parámetros de la función pulmonar estudiados y el tratamiento de la DM2, ajustada por los factores intervinientes se empleó la técnica de regresión lineal múltiple dado el carácter continuo de las variables dependientes.

\section{Resultados}

En total se tuvo información completa para 369 pacientes. No se encontraron diferencias estadísticamente significativas entre los tratados con $\mathrm{HO}$ o con insulina para variables como sexo, edad, talla o antecedentes de tabaquismo hipertensión arterial, infarto, falla cardiaca, asma, bronquitis crónica, enfisema, u otros antecedentes. De igual forma, no se observaron diferencias significativas

Tabla 1. Características basales de la población de ambos grupos de tratamiento ( $(\%)$ media $\pm s d$ ).

\begin{tabular}{|c|c|c|c|}
\hline Variable & $\begin{array}{c}\text { HO } \\
(n=306)\end{array}$ & $\begin{array}{l}\text { Insulina } \\
(\mathrm{n}=63)\end{array}$ & Valor $p$ \\
\hline Masculino & $148(48.37)$ & $34(53.97)$ & 0.419 \\
\hline $\begin{array}{l}\text { Edad } \\
\qquad \begin{array}{r}35-45 \\
46-55 \\
56-65\end{array}\end{array}$ & $\begin{array}{c}53.48 \pm 8.06 \\
59(19.28) \\
109(35.62) \\
138(45.10)\end{array}$ & $\begin{array}{c}53.37 \pm 8.20 \\
10(15.87) \\
28(44.44) \\
25(39.68)\end{array}$ & $\begin{array}{l}0.920 \\
0.847\end{array}$ \\
\hline $\mathrm{HbAlc}$ & $8.66 \pm 2.37$ & $9.17 \pm 2.15$ & 0.2 \\
\hline Mal control* & $219(71.57)$ & $51(80.95)$ & 0.126 \\
\hline Tiempo de diagnóstico & $8.19 \pm 6.32$ & $9.21 \pm 5.84$ & 0.239 \\
\hline Talla & $160.89 \pm 10.09$ & $160.71 \pm 9.71$ & 0.900 \\
\hline Peso & $73.48 \pm 14.00$ & $73.40 \pm 14.71$ & 0.968 \\
\hline \multicolumn{4}{|l|}{ Fuma } \\
\hline No fumador & $139(45.42)$ & $23(36.51)$ & 0.157 \\
\hline Fumador anterior & $124(40.52)$ & $28(44.44)$ & \\
\hline Fumador actual & $43(14.05)$ & $12(19.05)$ & \\
\hline Cigarrillos / día & $6.74 \pm 6.62$ & $4.25 \pm 5.14$ & 0.234 \\
\hline Mediana (p75-p25) & $4.0(2-10)$ & $3.0(2-4.5)$ & \\
\hline \multicolumn{4}{|c|}{$\begin{array}{l}\text { * Determinado de acuerdo con el estudio de Nathan DM; Buse JB; Davidson } \\
\text { MB; Ferrannini E et al. Diabetes Care : 2009; 32(1): 193-203. } \\
\text { HO: Hipoglicemiantes, HbAlc: hemoglobina glucosilada. }\end{array}$} \\
\hline
\end{tabular}


Tabla 2. Antecedentes patológicos por grupos de tratamiento. ( $(\%)$, media $\pm s d)$.

\begin{tabular}{|l|c|c|c|}
\hline Variable & $\begin{array}{c}\text { HO } \\
(\mathbf{n = 3 0 6})\end{array}$ & $\begin{array}{c}\text { Insulina } \\
(\mathbf{n}=\mathbf{6 3})\end{array}$ & Valor p \\
\hline Hipertensión & $112(36.60)$ & $17(26.98)$ & 0.145 \\
Infarto agudo al miocardio & $9(2.94)$ & $1(1.59)$ & 0.547 \\
Insuficiencia cardiaca & $2(0.65)$ & $0(0.00)$ & 0.521 \\
Eventos cerebrovasculares & $3(0.98)$ & $2(3.17)$ & 0.171 \\
Embolia pulmonar & $0(0.00)$ & $0(0.00)$ & - \\
Bronquitis crónica & $7(2.29)$ & $0(0.00)$ & 0.226 \\
Enfisema pulmonar & $1(0.33)$ & $0(0.00)$ & 0.367 \\
Asma bronquial & $9(2.94)$ & $1(1.59)$ & 0.547 \\
Exposición a polvo & $25(39.68)$ & $88(28.76)$ & 0.087 \\
Exposición a gases & $13(20.63)$ & $39(12.75)$ & 0.102 \\
Exposición a humo de leña & $20(31.75)$ & $79(25.82)$ & 0.334 \\
\hline HO: Hipoglucemiantes orales. & & & \\
\hline
\end{tabular}

Tabla 3. Función pulmonar por grupos de tratamiento* ( $(\%)$, media $\pm s d$ ).

\begin{tabular}{|c|c|c|c|}
\hline Variable & $\begin{array}{c}\text { HO } \\
(n=306)\end{array}$ & $\begin{array}{c}\text { Insulina } \\
(n=63)\end{array}$ & $\begin{array}{c}\text { Valor } \\
\mathbf{P}\end{array}$ \\
\hline VEF1 & $2.72 \pm 0.72$ & $2.67 \pm 0.75$ & 0.642 \\
\hline $\mathrm{CVF}$ & $3.41 \pm 0.93$ & $3.39 \pm 0.91$ & 0.851 \\
\hline VEF1/CVF & $80.39 \pm 6.53$ & $78.87 \pm 5.54$ & 0.086 \\
\hline VEF $1<70 \% *$ & $8(2.61)$ & $4(6.35)$ & 0.129 \\
\hline $\mathrm{CVF}<70 \% *$ & $6(1.96)$ & $3(4.76)$ & 0.190 \\
\hline VEF1/CVF<70\%* & $15(4.90)$ & $5(7.94)$ & 0.333 \\
\hline \multicolumn{4}{|c|}{$\begin{array}{l}\text { (*) Predicho por las ecuaciones de: Hankinson JL, Odencrantz JR, Fedan KB. } \\
\text { Spirometric reference values from a sample of the general US population. Am J } \\
\text { Respir Crit Care Med 1999; 159: 179-187. } \\
\text { HO. Hipoglucemiantes; VEF1: volumen espiratorio forzado en el primer segundo; } \\
\text { CVF: Capacidad Vital Forzada; VEF1/CVF: relación VEF1 /CVF; VEF1<70\%: valor } \\
\text { predicho menor al 70\% para VEF1; CVF1<70\%: valor predicho menor al 70\% para } \\
\text { CVF1; VEF1/CVF<70\%: valor predicho menor al 70\% para la relaciónVEF1/CVF. }\end{array}$} \\
\hline
\end{tabular}

entre los grupos con respecto a control o a duración de la diabetes (Tablas 1 y 2).

La Tabla 3 muestra los valores absolutos de VEF1, CVF y relación $\mathrm{VEF} 1 / \mathrm{CVF}$ en los grupos de tratamiento. Aunque el grupo de $\mathrm{HO}$ tuvo valores discretamente superiores de VEF1 y CVF, las diferencias no fueron estadísticamente significativas. De igual forma, tampoco se documentó diferencias en valores predichos menores a $70 \%$ para $\mathrm{CVF}$, VEF1 y relación VEF1/CVF, aunque estas proporciones tendieron a ser mayores en el grupo de insulina. En el análisis de residuales (diferencias entre los valores observados y los predichos), y después de ajustar por el potencial efecto de sexo, edad, IMC, HbAlc, tabaquismo, y tiempo desde el diagnóstico, ambos grupos se asociaron con menor función pulmonar que la predicha para VEF1, CVF, y la relación VEF1/CVF, pero con menores residuales promedios para el grupo de HO ( $\mathrm{P}<0.0001$, Tabla 4). Al evaluar niveles de marcadores inflamatorios en ambos grupos de tratamiento, no hubo cambio estadísticamente significativo en ninguno de los marcadores de inflamación entre los grupos de tratamiento (Tabla 5).

\section{Discusión}

Este estudio muestra que después de ajustar por determinantes conocidos de la función pulmonar (sexo, edad y talla, mediante ecuaciones de predicción), así como por

Tabla 4. Función pulmonar media residual (*) en diabéticos. Por grupo de tratamiento.

\begin{tabular}{|l|c|c|c|}
\hline Marcador & \multicolumn{1}{|c|}{$\begin{array}{c}\text { HO } \\
(\mathbf{n = 3 0 6})\end{array}$} & $\begin{array}{c}\text { Insulina } \\
(\mathbf{n = 6 3})\end{array}$ & $\begin{array}{c}\text { Valor } \\
\mathbf{p}\end{array}$ \\
\hline $\begin{array}{l}\text { PCR ultrasensible } \\
\text { (mgrs/dL) }\end{array}$ & $2.37 \pm 4.67$ & $2.82 \pm 4.12$ & 0.480 \\
$\begin{array}{l}\text { Ferritina } \\
\text { (Ugrs/L) }\end{array}$ & $148.69 \pm 135.19$ & $162.29 \pm 151.58$ & 0.477 \\
$\begin{array}{l}\text { Fibrinógeno } \\
\text { (mgrs/dL) }\end{array}$ & $437.14 \pm 115.16$ & $435.59 \pm 166.89$ & 0.922 \\
$\begin{array}{l}\text { IL-6 } \\
\text { (pg/mL) }\end{array}$ & $8.90 \pm 5.47$ & $8.48 \pm 4.10$ & 0.114 \\
$\begin{array}{l}\text { TNF- } \alpha \\
\text { (pg/mL) }\end{array}$ & $8.78 \pm 4.44$ & 0.875 \\
\hline $\begin{array}{l}\text { HO: Hipoglucemiantes orales, IL-6: interleuquina 6, TNF- } \alpha: \text { Factor de Necrosis } \\
\text { Tumoral, PCR: Proteína C reactiva. }\end{array}$ & \\
\hline
\end{tabular}

Tabla 4. Función pulmonar media residual (*) en diabéticos. Por grupo de tratamiento.

\begin{tabular}{|c|c|c|c|c|c|c|c|c|}
\hline \multirow[t]{3}{*}{ Variable } & \multicolumn{2}{|c|}{ Ho } & \multicolumn{2}{|c|}{ Insulina } & \multicolumn{3}{|c|}{ Diferencia } & \multirow{3}{*}{$\begin{array}{c}\text { Valor } \\
\mathbf{P}\end{array}$} \\
\hline & \multirow{2}{*}{$\mathbf{N}$} & \multirow{2}{*}{ Media } & \multirow{2}{*}{$\mathbf{N}$} & \multirow{2}{*}{ Media } & \multirow{2}{*}{ Media } & \multicolumn{2}{|c|}{ I.C $95 \%$} & \\
\hline & & & & & & LI & LS & \\
\hline rVEF1 & 306 & -135.5 & 63 & -193.2 & 57.598 & 32.45 & 82.74 & 0.005 \\
\hline $\mathrm{rCVF}$ & 306 & -202.1 & 63 & -247.7 & 45.6 & 20.84 & 70.39 & 0.023 \\
\hline rVEF1/CVF & 306 & 1.019 & 63 & 1.002 & 0.017 & 0.012 & 0.022 & $<0.0001$ \\
\hline
\end{tabular}

(*) Ajustado por diferencias en sexo, edad, índice de masa corporal, HbA1, tabaquismo, y tiempo de diagnóstico de la diabetes.

IC: Intervalo de confianza. LI: límite inferior; LS: límite superior. HO: Hipoglucemiantes orales; rVEF1: residual VEF1; rCVF: residual CVF; rVEF1/CVF: residual de la relación VEF1/CVF. 
tabaquismo, control de la diabetes mediante HbAlc, IMC, y por tiempo desde el diagnóstico de la DM2, la función pulmonar en los tratados con $\mathrm{HO}$ es mejor que la de los tratados con insulina, ya que se encontraron menores residuales promedios (diferencias entre valores observados y predichos) para CVF, VEF1y relación VEF1/CVF a favor de los HO. Por otro lado, no se observaron diferencias estadísticamente significativas en niveles de marcadores de inflamación entre aquellos tratados con insulina o con HO.

Nuestro estudio debe considerarse de naturaleza exploratoria, debido a las limitaciones inherentes a los estudios no experimentales, y especialmente al diseño de corte transversal. El sesgo de mala clasificación en el tiempo de duración de la diabetes es una posibilidad, debido a que se basó en la memoria de los sujetos para conocer los antecedentes médicos, así como también en la medición de la exposición al cigarrillo, dado que no contamos con una medida de ajuste como años-paquete. Debido a la naturaleza del estudio (corte transversal), esto también aplicaría para la mala clasificación de control de diabetes, ya que la medición y clasificación del mismo a través de la HbA1, se realizó en un solo momento en el tiempo, y no se puede saber antes de la medición cómo era el comportamiento. Esto pudo haber llevado a ajuste incompleto por el efecto de estas variables, confusión residual, y limitar las inferencias que se puedan derivar de este estudio. Sin embargo, si existió sesgo de mala clasificación en estas variables, tendería a ser no diferencial, puesto que fue igual para los dos grupos de tratamiento estudiados, y haber llevado el estudio principalmente hacia no encontrar las asociaciones detectadas en este estudio. Finalmente, nuestro estudio no cuenta con información del tratamiento previo de los sujetos, ni de las dosis que recibían de insulina o de los $\mathrm{HO}$, por lo que otros ajustes por estas variables no fueron posibles.

Es interesante especular por qué el tratamiento de la DM2 con insulina o con HO pudiese asociarse per se con diferencias en la función pulmonar, si éstas no estuvieran condicionadas por variables como obesidad, control glucémico y duración de la diabetes, que determinan en gran manera el tratamiento de la DM2 y a su vez se han asociado con peor función pulmonar en DM2 $(4,15,18)$. Se reconoce que el estado de resistencia a la insulina y de hiperinsulinismo compensatorio visto en sujetos con DM2, especialmente obesos, puede ser deletéreo para el endotelio humano (11), aunque sigue siendo un reto dilucidar un efecto del otro. Se ha documentado in vitro que la hiperinsulinemia compensatoria puede llevar a incremento en niveles de activador/inhibidor-1 del plasminógeno, endotelina, y de eventos proliferativos/mitogénicos a través de vías dependientes de la MAP-kinasa $(11,25)$. De igual forma, la resistencia a la insulina se ha asociado con deterioro de la función pulmonar en mujeres con DM2 (26). Podría ser interesante especular que insulina exógena en dosis altas, en presencia de estados de hiperinsulinismo compensatorio, pudiese también actuar a través del receptor para el factor de crecimiento-1 "insuline-like" (27) y promover deterioro de la función pulmonar por efecto mitogénico sobre el endotelio pulmonar.

Sin embargo, un mecanismo adicional que explicaría el deterioro asociado con el estado de resistencia a la insulina e hiperinsulinismo, es por disfunción de la musculatura del aparato pulmonar $(28,29)$. Este efecto explicaría el mayor deterioro sobre la CVF y no sobre el VEF1, y un patrón predominantemente restrictivo o hipodinámico $(3,30,31)$.

Adicionalmente, es posible que los $\mathrm{HO}$, especialmente aquellos sensibilizadores al efecto de la insulina como la metformina y las tiazolidinedionas, pudiesen disminuir el potencial daño pulmonar promovido por el estado de resistencia a la insulina y/o el hiperinsulinismo compensatorio en sujetos con DM2 con estas características (13). Nosotros evaluaremos esta atractiva hipótesis en este mismo grupo de pacientes, buscando diferencias en función pulmonar entre grupos de $\mathrm{HO}$ (sensibilizantes versus secretagogos), teniendo en cuenta el efecto modificador del riesgo de la obesidad como marcador indirecto de resistencia a la insulina. Estudios previos han mostrado niveles mayores de marcadores de inflamación sistémica, como ferritina (6) y PCR (33) en pacientes con diabetes tipo 2 y se han encontrado niveles elevados de mediadores de inflamación (IL-1, IL6, TNF $\alpha$ ) asociados con aumento en la resistencia a la insulina (34). Si los HO atenuaran la inflamación de baja intensidad vista en esta condición, se esperaría haber observado una diferencia entre los niveles de marcadores de inflamación en este estudio; sin embargo, este estudio no encontró cambio en estos marcadores entre tratamiento con insulina o HO, por lo que se asume que si los HO están asociados con mejor función pulmonar, el mecanismo puede no ser a través de atenuación de la inflamación, o que haya otros marcadores que pudieran ser responsables de la misma y que no fueron evaluados en este estudio. En conclusión, nuestro estudio muestra que existen diferencias en función pulmonar entre pacientes con DM2 que reciben tratamiento con $\mathrm{HO}$ o con insulina. Debido a las limitaciones expuestas, no es posible excluir que estas diferencias a su vez estén condicionadas por ajuste incompleto de variables cointervinientes como obesidad, control glucémico y duración de la diabetes. Sin embargo, es interesante postular que el tratamiento de la DM2 con insulina o con $\mathrm{HO}$ pudiese asociarse per se con diferencias en la función pulmonar. En un futuro, y en ausencia de experimentos clínicos controlados, estudios de cohortes con seguimiento a mediano y largo plazo podrían ser la mejor forma de diferenciar estos efectos.

\section{Referencias}

1. Davis T, Knuiman M, Kendall P, et al. Reduced pulmonary function and its associations in type 2 diabetes: the Fremantle Diabetes Study. Diab Res Clin Pract 2000; 50: 153-9.

2. Terzano C, Morano S, Ceccarelli D, Conti V, Paone G, Un Petroianni, et al. Effect of insulin on airway responsiveness in patients with type 2 diabetes mellitus: a cohort study. J Asthma 2009; 46: 703-7. 
3. Dennis R, Maldonado D, Rojas MX, Aschner P, Rondon M, Charry L, et al. Diabetes mellitus tipo 2 y deterioro de la función pulmonar. Acta Med Colomb 2008; 33: 105-10.

4. Yeh HC, Punjabi NM, Wang NY, Pankow JS, Duncan BB, Cox CE, et al. Cross-sectional and prospective study of lung function in adults with type 2 diabetes: the Atherosclerosis Risk in Communities (ARIC) study. Diabetes Care 2008; 31: 828-32.

5. Borst, BV; Gosker HR, Zeegers MP, Annemie M. W. J. Schols. Pulmonary function in diabetes: a metaanalysis. Chest 2010; 138: 393-440.

6. Ford ES, Cogswell ME. Diabetes and serum ferritin concentration among US adults. Diabetes Care 1999; 22: 1978-83.

7. Martins JO, Ferracini M, Anger DB, Martins DO, Ribeiro LF Jr, Sannomiya $\mathbf{P}$, Jancar S. Signaling pathways and mediators in LPS-induced lung inflammation in diabetic rats: role of insulin. Shock 2010; 33: 76-82.

8. Schmidt MI, Duncan BB, Sharret AR, Lindberg G, Savage PJ, Offenbacher S, Azambuja MI, Tracy RP, Heiss G. Markers of inflammation and prediction of diabetes mellitus in adults (Atherosclerosis Risk in Communities a tudy): a cohort study. Lancet 1999; 353: 1649-52.

9. Dahl M, Tybjaerg-Hansen A, Vestbo J, Lange P, Nordestgaard BG. Elevated plasma fibrinogen associated with reduced pulmonary function and increased risk of chronic obstructive pulmonary disease. Am J Respir Crit Care Med 2001; 164: $1008-11$.

10. Rodriguez-Moran M, Guerrero-Romero F. Increased levels of C reactive protein in non-controlled type II diabetic subjects. J Diabetic Complications 1999; 13: 211-215.

11. Montagnani M, Golovchenko I, Kim I, Koh GY, Goalstone ML, Mundhekar AN et al. Inhibition of phosphatidylinositol 3-kinase enhances mitogenic actions of insulin in endothelial cells. J Biol Chem 2002; 277: 1794-9.

12. Guazzi M, Brambilla R, De Vita S, Guazzi M D. Diabetes worsens pulmonary diffusion in heart failure, and insulin counteracts this effect. Am J Respir Crit Care Med 2002; 166: 978-82

13. Kim HJ, Lee JY, Jung HS, Kim DK, Lee SM, Yim JJ, et al. The impact of insulin sensitisers on lung function in patients with chronic obstructive pulmonary disease and diabetes. Int J Tuberc Lung Disease 2010; 14: 362-7

14. Lange P, Groth S, Kastrup J, Mortensen J, Appleyard M, Nyboe J, et al. Diabetes mellitus, plasma glucose and lung function in a cross-sectional population study. Eur Respir 1989; 2: 14-19.

15. McKeever TM, Weston PJ, Hubbard R, Fogarty A. Lung function and glucose metabolism: an analysis of data from from the third national health and nutrition examination survey. Am J Epidemiol 2005; 161: 546-56.

16. Walter RE, Beiser A, Givelber RJ, O'Connor GT, Gottlieb DJ. Association between glycemic state and lung function. Am J Respir Crit Care Med 2003; 167: 911-6.

17. Davis WA, Knuiman M, Kendall P, Grange V, Davis TME. Glycemic exposure is associated with reduced pulmonary function in type 2 diabetes. Diabetes Care 2004; $27:$ 752-7.

18. Dennis R, Maldonado D, Rojas MX, Aschner P, Rondón M, Charry L, et al. Inadequate glucose control in type 2 diabetes is associated with impaired lung function and, systemic inflammation: a cross-sectional study. BMC Pulmonary Medicine 2010, 10: 38

19. Asociación Latinoamericana de Diabetes. Guías ALAD 2000. Rev Asoc Latinoam Diab; supl 1: Edición extraordinaria, 2001.

20. American Thoracic Society. Lung function testing: selection of reference values and interpretative strategies. Am Rev Respir Dis 1991; 144: 1202-18.

21. Nathan DM, Buse JB, Davidson MB, Ferrannini E, Holman RR, Sherwin $\mathbf{R}$, et al. Medical management of hyperglycemia in type 2 diabetes: a consensus algorithm for the initiation and adjustment of therapy: a consensus statement of the american diabetes association and the european association for the study of diabetes. Diabetes Care 2009; 32: 193-203.

22.Hankinson JL, Odencrantz JR, Fedan KB. Spirometric reference values from a simple of the general US population. Am J Respir Crit care Med 1999; 159: 179-187.

23. Rojas MX, Dennis R. Valores de referencia para parámetros espirométricos en la población adulta residente en Bogotá, DC, Colombia. Biomédica 2010; 30: 82-94.

24. Rodriguez N, Rojas MX, Guevara DP, Dennis RJ, Maldonado D. Generación de valores de referencia para la evaluación de la espirometria: Estudio en una población Colombiana. Acta Med Colomb 2002; 27: 389-97.

25. Goalstone ML, Carel K, Leitner J, Draznin B. Insulin stimulates the phosphorylation and activity of farnesyltransferase via the ras-mitogen-activated protein kinase pathway. Endocrinology 1997; 138: 5119-24.

26.D Lawlor D, Ebrahim S, Davey S. Association of birth weight with adult lung function: findings from the British Women's Heart and Health Study and a meta-analysis. Diabetología 2004; 47: 195-203.

27. Sowers, JR. Insulin and Insulin-Like Growth Factor in Normal and Pathological Cardiovascular Physiology; Hypertension 1997; 29: 691-9.

28.Lazarus, R, Sparrow D, and Weiss ST. Handgrip strength and insulin levels: Cross-sectional and prospective associations in the normative aging study. Metabolism 1997; 46: 1266-9.

29. Barzilay JI, Cotsonis GA, Walston, J, Schwartz, AV, Satterfield S, Miljkovic I, et al. Insulin resistance is associated with decreased quadriceps muscle strength in nondiabetic adults aged $>70$ years. Diabetes Care 2009; 32: 736-8.

30.Maccioni FJ, Colebatch JH. Lung volume and distensibility in insulindependent DM. Am rev Respir Dis 1991; 143: 1253-6.

31. Fehrenbach H, Kasper M, Tshernig T, Shearman MS, Schuh D, Müller M. Receptor for advanced glycation end products exhibits highly differential cellular and subcellular localization in rat and human lung. Cell Mol Biol 1998; 44: 1147-57.

32. Vracko R, Thorning D, Huang TW. Basal lamina of alveolar epithelium and capillaries: cuantitative changes with aging and in diabetes mellitus. Am Rev Respir Dis 1979; 120: 973-83.

33. Dahl M, Vestbo G, Lange P, Bojesen SE, Tybjaerg-Hansen A, Nordestgaard BG. C-reactive Protein as a predictor of prognosis in chronic obstructive pulmonary disease. Am J Respir Crit Care Med 2007; 175: 250-5.

34. Bronwlee M. Biochemistry and molecular cell biology of diabetic complications. Nature 2001; 414: 813-20. 